\title{
5.4 W cladding-pumped Nd:YAG silica fiber laser
}

\author{
S. Yoo, A. S. Webb, R. J. Standish, T. C. May-Smith, J. K. Sahu* \\ Optoelectronics Research Centre, University of Southampton, Southampton, SO17 1BJ, United Kingdom \\ *jks@orc.soton.ac.uk
}

\begin{abstract}
We report on the spectroscopy and laser characteristics of Nd-doped fiber, fabricated by rod-in-tube from Nd:YAG as a core material with silica cladding. A cladding-pumped CW laser operation at $1058 \mathrm{~nm}$ with $52 \%$ slope-efficiency is demonstrated.

OCIS codes: (060.3510) Lasers, Fiber; (060.2290) Fiber materials.
\end{abstract}

\section{Introduction}

In recent years, the dramatic progress in high power fiber lasers has generated significant interest in utilizing different host materials and novel fiber fabrication techniques to manipulate the spectroscopic properties in rareearth (RE) doped fibers, such as high gain characteristics, reduced photodarkening. Moreover, high RE ion concentration is required to minimize the fiber length and improve pump efficiency in cladding-pumped configuration whilst maintaining sufficient pump absorption. Traditionally, high power fiber sources use silica as the host glass, which has limitations on the level of RE ions that can be incorporated without clustering effects. This restricts the device length for high power laser and amplifier applications, and thereby reduces the threshold for undesirable non-linear effects in the fiber. Moreover, the limited choices of co-dopants that can be incorporated into the silica matrix restricts the optical properties of RE-doped fibers. Recently, fibers with core material drawn from an Er:YAG or Yb:YAG crystal and silica cladding have been demonstrated [1,2], but to the best of our knowledge no reports on high power lasers are available. Here we present spectroscopy and laser characteristics of a Nd-doped non-silica core and silica cladding fiber, fabricated using a rod-in-tube technique and a Nd:YAG crystal rod as core material. The fiber generated $5.4 \mathrm{~W}$ of continuous-wave (cw) laser output power with $52 \%$ slope efficiency when cladding pumped at $808 \mathrm{~nm}$.

\section{Experiments and results}

A Nd:YAG crystal rod of $2.5 \mathrm{~mm}$ in diameter was inserted in the centre of a drilled silica rod (Heraeus F300) of OD $24 \mathrm{~mm}$. The resultant preform was drawn into $200 \mu \mathrm{m}$ inner-cladding diameter fiber with a $20 \mu \mathrm{m}$ core and coated with a low index polymer outer cladding, producing a nominal inner-cladding numerical aperture (NA) of 0.48. Fig. 1 shows the RI profile of the fiber. The core NA is 0.47. Fig. 2 shows the line-scan profiles of elemental Si, Al and $\mathrm{O}$ across the core and cladding regions in fiber, measured using Energy Dispersive X-ray Spectroscopy (EDX) with SEM image of the fiber cross-section inset. Note that the Nd-concentration in the core was below the detection limit of the instrument.

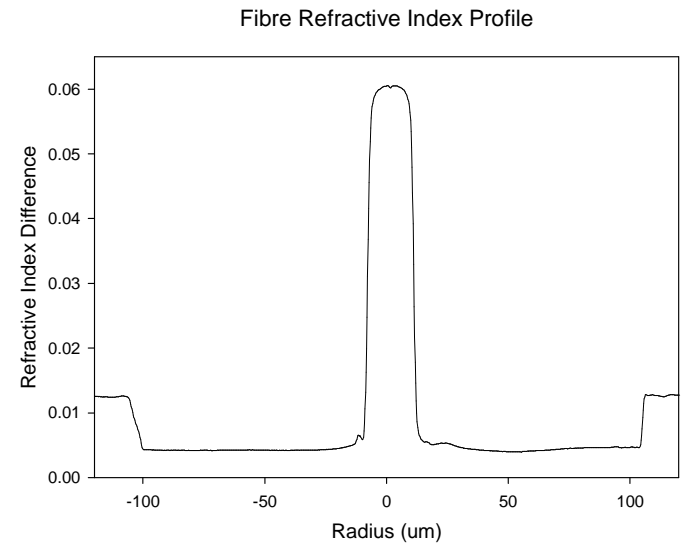

Fig. 1 Refractive index profile of Nd:YAG core-silica clad fiber

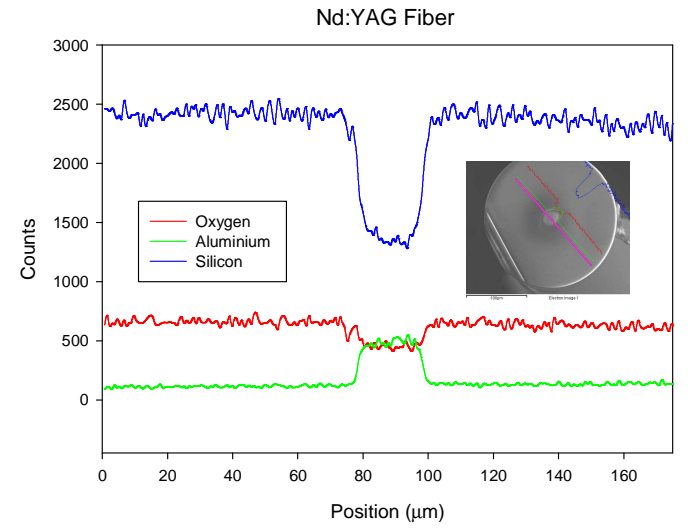

Fig. 2 EDX data of fabricated fiber with SEM image of fiber crosssection (inset) 
Fig. 3 shows the Nd absorption spectrum in fiber, measured using a white-light source and OSA. Three broad absorption bands centered at around $742 \mathrm{~nm}, 803 \mathrm{~nm}$ and $870 \mathrm{~nm}$ were observed. The background loss in the fiber, measured using a high resolution OTDR at $1280 \mathrm{~nm}$, is $1.2 \mathrm{~dB} / \mathrm{m}$. We have measured the fluorescence lifetimes in, $\mathrm{Nd}$ :YAG bulk crystal, fiber drawn from Nd:YAG core material, and Nd:Al-doped silica fiber fabricated in-house using conventional MCVD (modified chemical vapour deposition)-solution doping technique, as $260 \mu \mathrm{s}, 330 \mu \mathrm{s}$, and $490 \mu \mathrm{s}$, respectively. The neodymium lifetime in YAG based fiber lies between the Nd:YAG crystal and $\mathrm{Nd}: \mathrm{Al}$ fiber. We calculated the emission cross-section in the Nd:YAG fiber from the Fuchtbauer-Ladenburg relation. The peak emission cross-section at $1058 \mathrm{~nm}$ is $1.9 \times 10^{-24} \mathrm{~m}^{2}$. The broad emission spectrum in the fiber (not presented here) indicates the amorphous nature of the Nd:YAG core after fiber drawing.

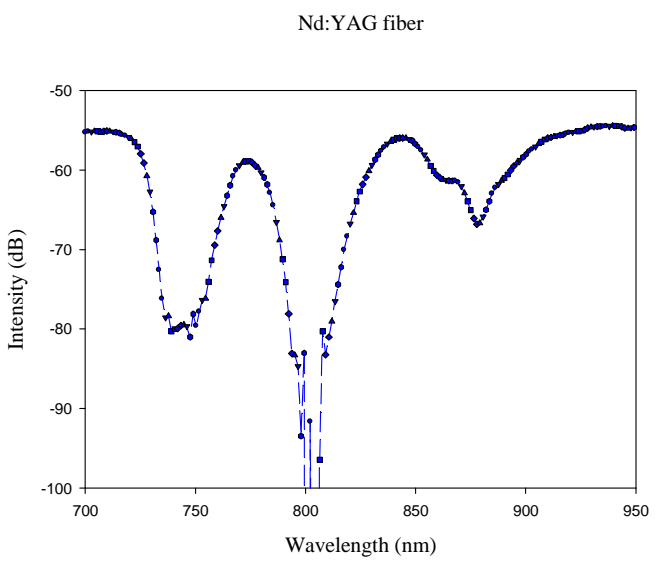

Fig. 3. White light absorption in a $2 \mathrm{~m}$ long fiber.

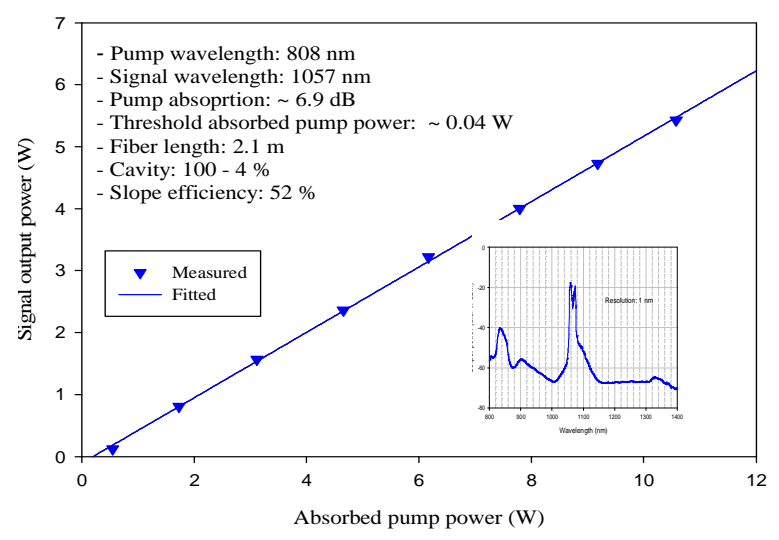

Fig.4. Fiber laser output power and laser spectrum at maximum output power (OSA resolution $1 \mathrm{~nm})$

Laser efficiency was tested in cladding-pumped configuration using a fiber coupled $808 \mathrm{~nm}$ laser diode with maximum output power of $20 \mathrm{~W}$. The pump beam was launched into the fiber via a combination of optics, including lenses and a dichroic mirror with high transmission at the signal wavelength and high reflection at the pump wavelength. The pump input end of the fiber was cleaved perpendicularly to the fiber axis to provide a Fresnel reflection for the laser cavity. The other end of the fiber was angle cleaved. A dichroic mirror with high reflection at signal wavelength provided $100 \%$ signal feedback to the fiber via the aspheric lens. The laser output was collected from the pump launched end. The fiber length was varied to maximize the signal output power. The laser characteristics are shown in Fig. 4, together with the spectrum taken at maximum output power. The maximum output power of $5.4 \mathrm{~W}$ was limited by available pump power. We achieved $52 \%$ of slope efficiency with respect to absorbed pump power for a $2.1 \mathrm{~m}$ long fiber. The laser emission was centered at wavelength $1058 \mathrm{~nm}$.

\section{Conclusion}

A Nd-doped yttrium-aluminium rich core and silica cladding fiber is demonstrated. The broad neodymium absorption and emission spectra observed in the fiber implies that the Nd:YAG crystal used in perform core has become amorphous after fiber drawing. Cladding-pumped operation in this fiber exhibited a good laser efficiency, showing the potential of this core composition for high power generation. We are investigating the pulse operation in this fiber and the work will be presented in conference.

\section{References}

[1] J. Ballato, T. Hawkins, P. Foy, B. Kokuoz, R. Stolen, C. McMillen, M. Daw, Z. Su, T. M. Tritt, M. Dubinskii, J. Zhang, T. Sanamyan, and M. J. Matthewson, "On the fabrication of all-glass optical fibers from crystals," J. Appl. Phys. 105, 053110 (2009).

[2] C. -C. Lai, K..-Y. Huang, H. -J. Tsai, K. -Y. Hsu, S. -K. Liu, C. -T. Cheng, K. -D. Ji, C. -P. Ke, S. -R. Lin, and S. -L. Huang, "Yb ${ }^{3+}: Y_{A G}$ silica fiber laser," Opt. Lett. 34, 2357-2359 (2009). 